\title{
Electrically Tunable Collective Response in a Coupled Micromechanical Array
}

\author{
Eyal Buks and Michael L. Roukes
}

\begin{abstract}
We employ optical diffraction to study the mechanical properties of a grating array of suspended doubly clamped beams made of $\mathrm{Au}$. The device allows application of electrostatic coupling between the beams that gives rise to formation of a band of normal modes of vibration (phonons). We parametrically excite these collective modes and study the response by measuring the diffraction signal. The results indicate that nonlinear effects strongly affect the dynamics of the system. Further optimization will allow employing similar systems for real-time mechanical spectrum analysis of electrical waveforms.

[756]
\end{abstract}

Index Terms-Grating, MEMS, nonlinear, parametric resonance.

\section{INTRODUCTION}

$\mathbf{T}$ HE FIELD of microelectromechanical systems (MEMS) is forcing a profound redefinition of the nature and attributes of electronic devices. MEMS are created by leveraging microelectronic fabrication techniques to realize microstructures with full, three-dimensional relief. This technology allows motion to be incorporated into the function of microscale devices. These additional mechanical degrees-of-freedom now enable a wide variety of intriguing new applications (see for example [1]). Such advances now make it feasible to envisage new classes of signal processors, sensors, and even computational systems, with functionality derived primarily from their mechanical, rather than their electrical degrees of freedom. However, the design of such mechanical systems may be quite challenging due to nonlinear effects that may strongly affect the dynamics, as was demonstrated recently with individual MEMS resonators (see, for example, [2]). Moreover, integrating individual mechanical elements to realize large-scale systems requires developing methods to couple individual mechanical elements to facilitate new types of coordinated, collective and engineered system response. To date, coupling of only several micromechanical elements has been reported; nonetheless this makes possible micromechanical filters with programmed passband response at radio frequencies [3]. However, the collective response of large arrays of coupled micromechanical devices has not yet been achieved. In this work we report the first realization of a large array of coupled micromechanical

Manuscript received September 17, 2001; revised February 5, 2002. This work was supported by DARPA MTO/MEMS under Grant DABT63-98-10012. The work of E. Buks was supported by the Rothschild fellowship and the R. A. Millikan postdoctoral fellowship at Caltech. Subject Editor R. T. Howe.

E. Buks is with the Department of Electrical Engineering, Technion, Haifa 32000, Israel (e-mail: eyal@ee.technion.ac.il).

M. L. Roukes is with Condensed Matter Physics 114-36, California Institute of Technology, Pasadena, CA 91125 USA (e-mail: roukes@ caltech.edu).

Digital Object Identifier 10.1109/JMEMS.2002.805056 systems. This represents one of the first steps to large-scale integration of micromechanical devices.

Our device consists of an array of doubly clamped beams forming a diffraction grating. Similar devices were employed before as optical switches where the efficiency of diffraction is controlled by moving the grating with respect to the substrate beneath it, allowing thus optical modulation [4]-[6]. In the present work, however, we introduce coupling between the beams and we study the resultant dynamics of the system.

The devices described in this work are composed of 67 doubly clamped beam resonators. With inter-device mechanical coupling effectively absent, we first characterize each resonator separately and completely through a novel technique [7], [8]. Then, upon application of a controlled and tunable electrostatic interaction between the individual mechanical elements, mechanical coupling between them is induced and a collective spectrum of vibrational modes emerges. These collective modes of the coupled system are excited parametrically, and we employ fiber-optic based optical diffraction to study the induced response. A simple model describing our system predicts the generalized overall features of this response, but with only modest drive levels a rich and complex nonlinear spectrum emerges. We conclude our discussion describing the unique prospects these systems offer for spectral analysis of complex mechanical stimuli, and for real-time spectral analysis of electrical waveforms.

\section{FABRICATION AND CHARACTERIZATION}

The bulk micromachining process employed for sample fabrication is described in Fig. 1. In this process the substrate beneath the grating is completely etched away, thus allowing optical access to the grating from both sides. In the first step chemical vapor deposition is employed to deposit a 70-nm-thick layer of low-stress silicon nitride on the front and back sides of a $\mathrm{Si}$ wafer. A square window is opened in the silicon nitride on the back side using photolithography and wet etching [see Fig. 1(a)]. The structure shown in Fig. 1(b) is realized through a highly selective, anisotropic $\mathrm{KOH}$ etch for the backside of the Si wafer. This occurs within the patterned region and yields a $270-\mu \mathrm{m}$-square silicon nitride membrane on the front side of the wafer. The grating beams and adjacent electrodes are fabricated on top of this membrane using electron beam lithography, followed by thermal evaporation of Au and liftoff [see Fig. 1(c)]. Each resulting beam has length $b=270 \mu \mathrm{m}$, width $1 \mu \mathrm{m}$ and thickness $0.25 \mu \mathrm{m}$ (measured using an atomic force microscope) and the grating period is $a=4 \mu \mathrm{m}$. In the final step the membrane is removed using electron cyclotron 
$\mathrm{Au} \quad$ (a)

(e)

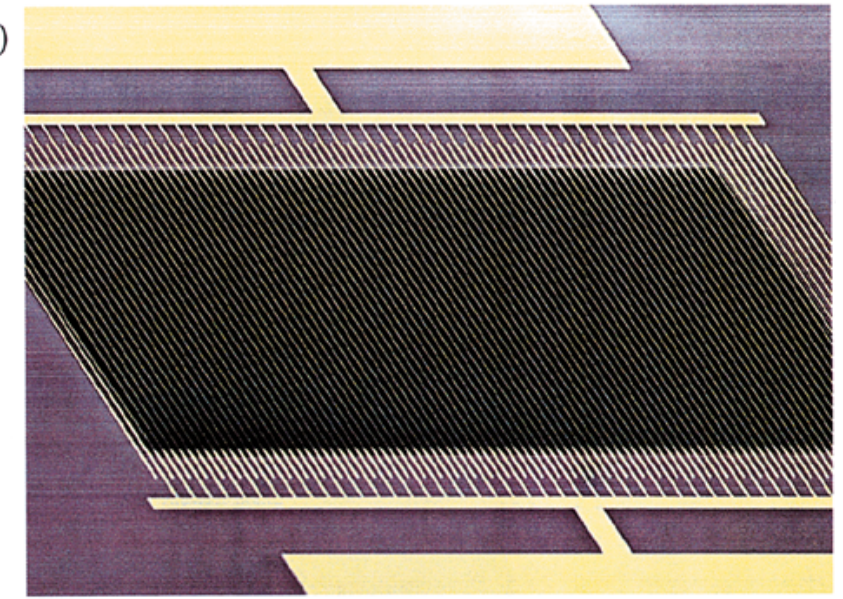

Fig. 1. The device is fabricated using bulk micromachining techniques. In steps (a) and (b), a suspended membrane of silicon nitride is formed. (c) A gold beam is fabricated on top of the membrane and (d) the membrane is etched, leaving the beam suspended. A side view micrograph of the device is seen in (e).
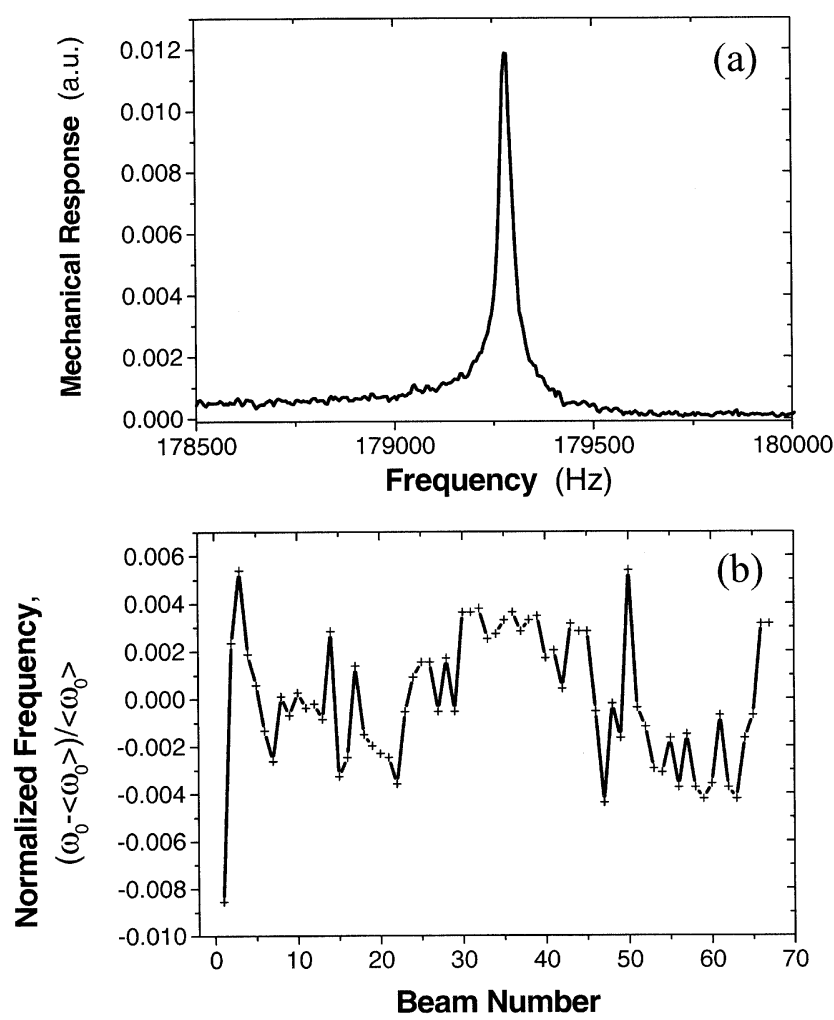

Fig. 2. (a) Response peak of an individual beam with a resonance frequency of $179.28 \mathrm{KHz}$. (b) Normalized resonance frequencies of all beams in the array $\left(\omega_{0}-\left\langle\omega_{0}\right\rangle\right) /\left\langle\omega_{0}\right\rangle$ where $\left\langle\omega_{0}\right\rangle$ is the average value.

resonance (ECR) plasma etching from the back side of the sample. This process step employs an $\mathrm{Ar} / \mathrm{NF}_{3}$ gas mixture, and results in suspension of the Au beam array [see Fig. 1(d)]. Fig. 1(e) shows a side view micrograph of the device. The

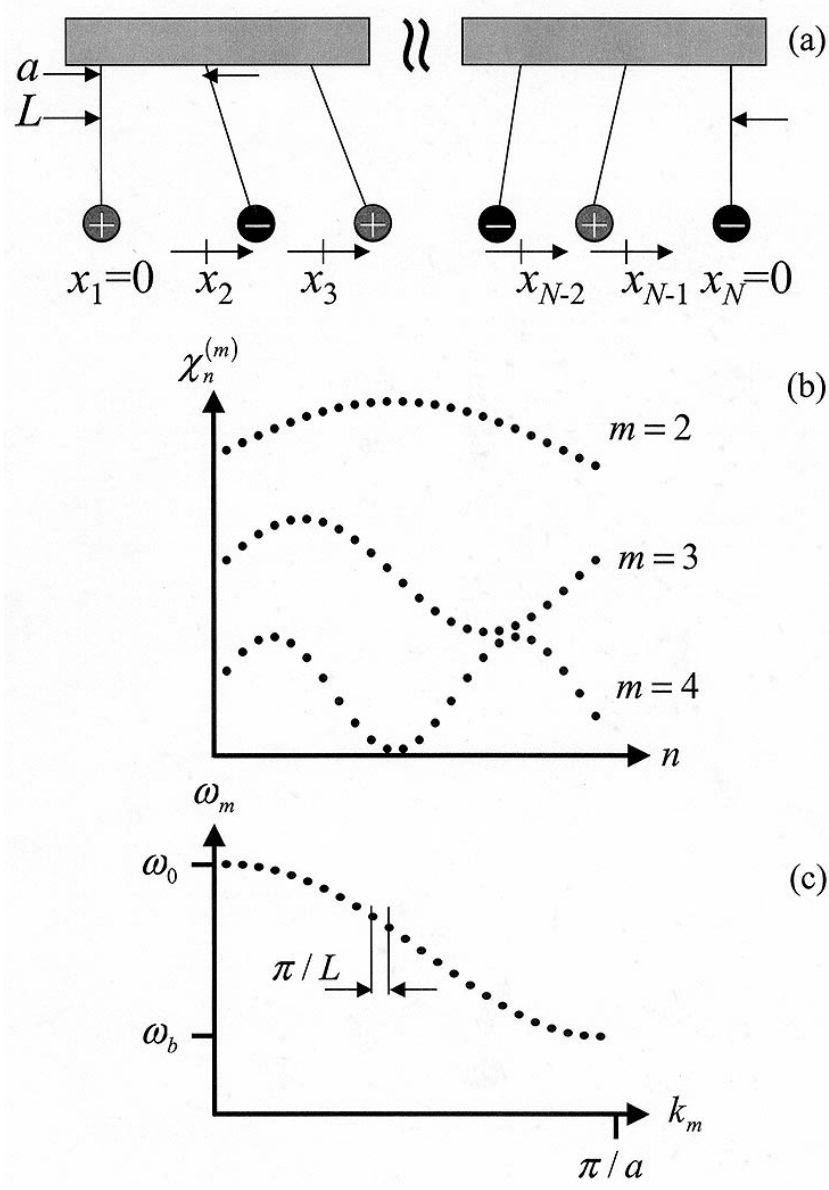

Fig. 3. (a) A model of $N$ coupled pendulums. (b) The shape of the three lowest modes. (c) The dispersion relation between the frequency $\omega_{m}$ of each mode and the wave vector $k_{m}$.

electrodes form two interdigitated combs; with fingers alternately connected to the two base electrodes. This design allows application of electrostatic forces between the beams.

To characterize uniformity within the device we measure the fundamental resonance frequency of each suspended beam in the array. This is done in situ, using the output from a commercial scanning electron microscope's imaging system to detect mechanical displacement. We have employed this technique previously to study the mechanical properties of individual, similarly fabricated Au beams [7], [8]. Fig. 2(a) shows a typical response peak from an individual beam. By measuring all 67 suspended beams in the array we find that the distribution of resonance frequencies has an average of $179.3 \mathrm{kHz}$ and a standard deviation of $0.53 \mathrm{kHz}$ [see Fig. 2(b)]. Individual mechanical quality factors $Q$ range from 2000 to 10000 . Note that no correlation is found between the location of the beam within the device and its specific resonance frequency or $Q$; the small beam-to-beam variations appear to be random.

\section{Modeling}

What is expected when a voltage $V$ is applied between the two combs? We employ a simple one-dimensional model for an $N$-element array of coupled pendulums [9] to describe our system [see Fig. 3(a)]. While the first and last pendulums in the array are clamped and stationary, all others $(n=2,3, \ldots, N-$ 
1) are free to oscillate about their equilibrium positions $n a$. Here $a$ represents the equilibrium spacing between neighboring pendulums. In the absence of any coupling, the angular frequency for small oscillations of each (identical) pendulum is $\omega_{0}$. The displacement of the system is described by a set of coordinates $x_{n}(n=1,2, \ldots, N)$ [see Fig. 3(a)]. Applying a voltage $V$ gives rise to an attractive interaction between each pendulum and its nearest neighbors $\varphi(s)=-C(s) V^{2} / 2$, where $s$ is the distance between the interacting pendulums and $C$ is the capacitance. Neglecting coupling between nonneighboring pendulums, and assuming small oscillations, we find the following set of equations of motion:

$$
m \ddot{x}_{n}=-m \omega_{0}^{2} x_{n}+u\left(2 x_{n}-x_{n-1}-x_{n+1}\right)
$$

where $n=2,3, \ldots, N-1$, and $u=-\varphi^{\prime \prime}(a)$ (here dots represents time derivatives and primes represent spatial derivatives). Note that $u>0$ due to the attractive nature of the interaction between nearest neighbors. These equations can be greatly simplified by employing a transformation to the eigenmodes (phonons) of the system

$$
x_{n}=\sum_{m=2}^{N-1} \chi_{n}^{(m)} v_{m}
$$

where $\chi_{n}^{(m)}=\sqrt{2 /(N-1)} \sin \left(k_{m}(n-1) a\right)$ is the spatial shape of mode number $m(m=2,3, \ldots N-1), k_{m}=(m-$ 1) $\pi / L$ is the wavevector, and $L=(N-1) a$ is the length of the system. Fig. 3(b) shows the shape of the three lowest modes $m=2,3,4$. Substituting (2) in (1) leads to a set of decoupled equations of motion:

$$
\ddot{v}_{m}=-\omega_{m}^{2} v_{m}
$$

where $\omega_{m}^{2}(V)=\omega_{0}^{2}-\left(2 C^{\prime \prime}(a) / m\right) V^{2} \sin ^{2}\left(k_{m} a / 2\right)$. A stationary voltage $V=V_{\mathrm{dc}}$ thus gives rise to the formation of a band of collective modes between frequencies $\omega_{0}$ and $\omega_{b}=\sqrt{\omega_{0}^{2}-\left(2 C^{\prime \prime}(a) / m\right) V_{d c}^{2}}$. The associated wavevectors, $k_{m}$, vary from $\pi / L$ to $\pi / a$ [see Fig. 3(c)].

Each mode can be selectively excited by adding an ac voltage to the dc bias, namely, $V=V_{\mathrm{dc}}+V_{\mathrm{ac}} \cos (\gamma t)$. Assuming $V_{\text {ac }} \ll V_{\text {dc }}$ we find from (3):

$$
\ddot{v}_{m}=-\omega_{m}^{2}\left(V_{d c}\right)\left(1-h_{m} \cos (\gamma t)\right) v_{m}
$$

where $h_{m}=2\left(V_{\mathrm{ac}} / V_{\mathrm{dc}}\right)\left[\left(\omega_{0} / \omega_{m}\right)^{2}-1\right]$. Thus, an ac voltage component gives rise to parametric excitation of each mode with amplitude $h_{m}$ [10]-[12]. Parametric resonance occurs when the frequency of the ac voltage $\gamma$ is close to $2 \omega_{m} / l$, where $l$ is an integer. Near these values the system may exhibit unstable behavior in which the amplitude of oscillations grows as a function of time. In the linear theory of parametric resonance this growth is exponential, and occurs when the amplitude of parametric excitation $h$ exceeds a critical value dependent upon the damping in the system. Most systems, however, possess some degree of nonlinearity which comes into play as soon as the amplitude of the motion becomes appreciable. Thus, while the linear theory is useful in determining the conditions for the occurrence of parametric resonance, it is inadequate for determining the steady-state response of the system. Unfortunately, the nonlinear coefficients of our system are not known and therefore its steady-state response cannot be predicted. However, we expect that the response of modes with high index $m$ will be relatively large because $h_{m}$ increases in magnitude with $m$.

We detect the collective mechanical vibrations of the array by diffraction measurements. With spatially uniform light incident upon the array, the intensity of diffraction is proportional to the form factor $\left|\phi\left(q_{x}\right)\right|^{2}$ where

$$
\phi\left(q_{x}\right)=\sum_{n=1}^{N} \exp \left[i q_{x}\left(n a+x_{n}(t)\right)\right] .
$$

Here, $q_{x}$ is the $x$ component of the change in wavevector between incoming and outgoing waves (the $x$ direction lies in the plane of the sample and is perpendicular to the long axis of the beams) [13]. Consider the case where the system is tuned to a diffraction peak, namely $q_{x} a=2 \pi l$ with $l$ an integer. As shown below, for this particular case the interpretation of the experimental results becomes greatly simplified. We calculate the form factor for the case where mode $m$ oscillates with amplitude $A_{m}$ and all other modes are stationary. Assuming small oscillations, namely, $q_{x} x \ll 1$, we find

$$
\begin{aligned}
\left|\phi\left(q_{x}\right)\right|^{2}=N^{2} & \left\{1-(1 / 2)\left(q_{x} A_{m}\right)^{2} \cos ^{2}\left(\omega_{m} t\right)\right. \\
\cdot & {\left.\left[1-\cot ^{2}\left(k_{m} a / 2\right)\left((-1)^{m}+1\right) / N^{2}\right]\right\} . }
\end{aligned}
$$

Thus, the collective mechanical oscillations give rise to an oscillatory component in the diffraction signal at angular frequency $2 \omega_{m}$. Note that the term proportional to $\cot ^{2}\left(k_{m} a / 2\right)$ is negligibly small unless $m$ is small.

\section{EXPERIMENTAL RESULTS AND DISCUSSION}

The optical setup utilized for the diffraction measurements is schematically depicted in Fig. 4. A polarization maintaining single mode fiber (numerical aperture NA $=0.15$ and core diameter $d=9 \mu \mathrm{m}$ ) delivers infrared light from a laser to the sample. We use a tunable wavelength diode laser operating in the range from 1535 to $1635 \mathrm{~nm}$. A spherical lens (focal length $f=1 \mathrm{~mm}$ ) collimates the beam, which illuminates the back side of the grating at normal incidence with respect to the array plane. At the array plane, located a distance $s_{1}=3$ $\mathrm{cm}$ from the collimator, we estimate the diameter of the beam to be $2 f \mathrm{NA}+s_{1} d / f=570 \mu \mathrm{m}$. For the results presented, the polarization of the incident light is TE, i.e., the electric field is parallel to the grating lines. We note, however, that similar results (not presented here) were obtained with perpendicular polarization. For the present case there are four diffraction peaks with angles $\theta_{n}=\sin ^{-1}(n \lambda / a)(n= \pm 1, \pm 2)$ with respect to the normal incidence. Here $\lambda$ is the wavelength of incident light. Transmitted light is collected by a cylindrical lens and focused into a second, single-mode fiber. The lens enables the intensity of collected light to be maximized without degrading the spectral resolution. The fiber delivers light collected from the first-order $(n=1)$ diffraction peak to a photodiode detector. The distance between the sample and the lens, $s_{2}=1 \mathrm{~cm}$, was chosen as a compromise between two conflicting considerations, namely, simultaneously 


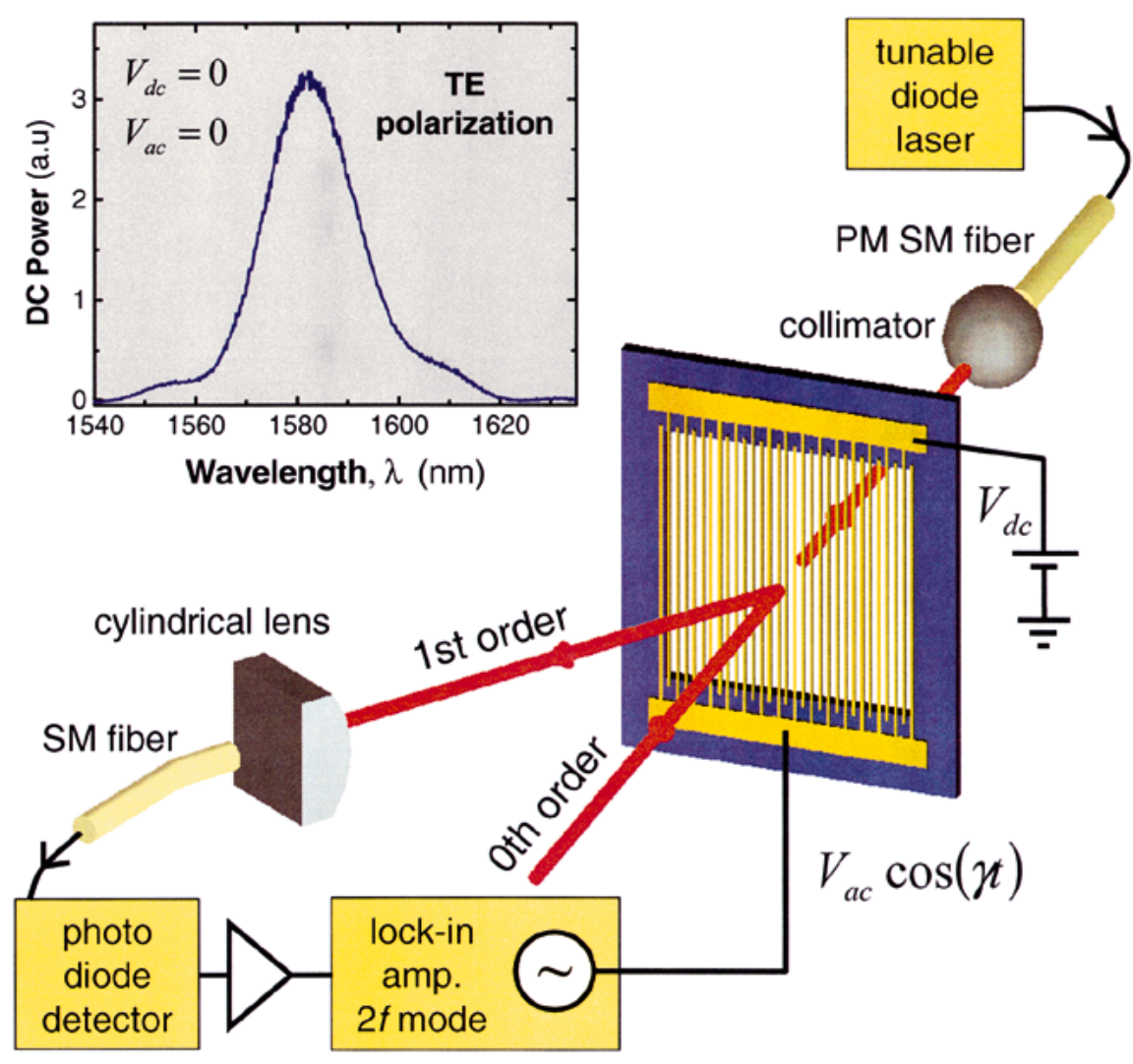

Fig. 4. The optical setup for measurements of the 1st order diffraction peak of the grating. Optical fibers are employed to deliver light to and from the vacuum chamber where the sample is mounted. The dc voltage $V_{\mathrm{dc}}$ introduces coupling between the beams and the ac voltage $V_{\mathrm{ac}}$ is used to parametrically excite the modes of vibration. A lock-in amplifier is employed to measure the response. The inset shows the dc signal of the photodetector as a function of wavelength (for $\left.V_{\mathrm{dc}}=V_{\mathrm{ac}}=0\right)$.

maximizing spectral resolution and light intensity. While the former consideration favors a large distance, the later favors a small one. For the distance chosen, the spectral resolution is $\delta \lambda=a \delta \theta \cos \theta_{1}=a\left(d / s_{2}\right) \cos \theta_{1} \simeq 3 \mathrm{~nm}$. All measurements are done at room temperature in vacuum of $\simeq 10^{-3}$ torr.

We first study diffraction from the array in the absence of any interelectrode bias voltage. The inset of Fig. 4 shows the intensity detected by the photo diode as a function of $\lambda$. The full-width at half-maximum (FWHM) of the diffraction peak is estimated using the Fraunhofer diffraction formula [13] to be $\delta \lambda \simeq \lambda / N=23 \mathrm{~nm}$. We find good agreement between this estimate and the measured value. No effect is resolved from the thermally driven mechanical vibrations of the beams. Their effect upon diffraction can be characterized by multiplying the diffracted intensity by a Debye-Waller factor, $\exp (-2 W)[14]$, [9]. In the present case we estimate that $2 W \simeq 10^{-8}$, hence thermal fluctuations are not expected to affect the diffraction from our micromechanical array significantly. This is confirmed by the experimental data.

In order to excite the modes of vibrations of the system externally, we apply a voltage $V=V_{\mathrm{dc}}+V_{\mathrm{ac}} \cos (\gamma t)$ between either side of the interdigitated electrode arrays. We tune the laser wavelength to the diffraction peak $(\lambda=1582 \mathrm{~nm})$ and measure the photodetector response using a lock-in amplifier operating in $2 f$ mode. This allows us to detect the Fourier component of the array response at angular frequency $2 \gamma$. Fig. 5 is a color map plot showing this second harmonic response, $R$, as a function of both $V_{\mathrm{dc}}$ and $f=\gamma / 2 \pi$. The amplitude of the ac voltage is $V_{\mathrm{ac}}=50 \mathrm{mV}$ for these measurements.

With $V_{\mathrm{dc}}=0$ we find a peak in $R$ at $f=179.3 \mathrm{kHz}$, associated with the fundamental frequency of the decoupled beams. The FWHM of the peak is $0.6 \mathrm{kHz}$, close to the standard deviation found in the distribution of the measured fundamental frequencies. This leads us to the conclusion that the width of the response peak at $V_{d c}=0$ is dominated by inhomogeneous broadening caused by the nonuniformity of the array.

As we increase $V_{\mathrm{dc}}$ we observe a gradual increase in the frequency range where relatively large response is observed; we associate this with the formation of a band of collective modes. The lower frequency bound of this range $f_{b}=\omega_{b} / 2 \pi$ (the bottom of the band) for relatively small $V_{\mathrm{dc}}$ is given theoretically by $f_{b}=\left(\omega_{0} / 2 \pi\right)\left(1-C^{\prime \prime}(a) V_{\mathrm{dc}}^{2} / m \omega_{0}^{2}\right)$. A least squares fit to the measured data (see dashed line in Fig. 5) yields $C^{\prime \prime}(a) / m \omega_{0}^{2}=$ $2.7 \times 10^{-4} \mathrm{~V}^{-2}$. For comparison we derive below a rough order of magnitude estimate of this factor. We substitute $m$, which should represent an effective mass of each beam, by the actual mass and we use the approximation $C^{\prime \prime}(a) \simeq \varepsilon_{0} b / g^{2}$ (assuming the case of parallel thin wires), where $\varepsilon_{0}$ is permittivity of free space and $g$ is the gap between neighboring beams. These crude approximations yield a value of $1.6 \times 10^{-4} \mathrm{~V}^{-2}$ which is quite close to the value deduced from the experimental data. The upper frequency bound of the band, on the other hand, shows some discrepancy with theory. While the measured value depends on $V_{\mathrm{dc}}$, our simple model predicts a upper value that is 


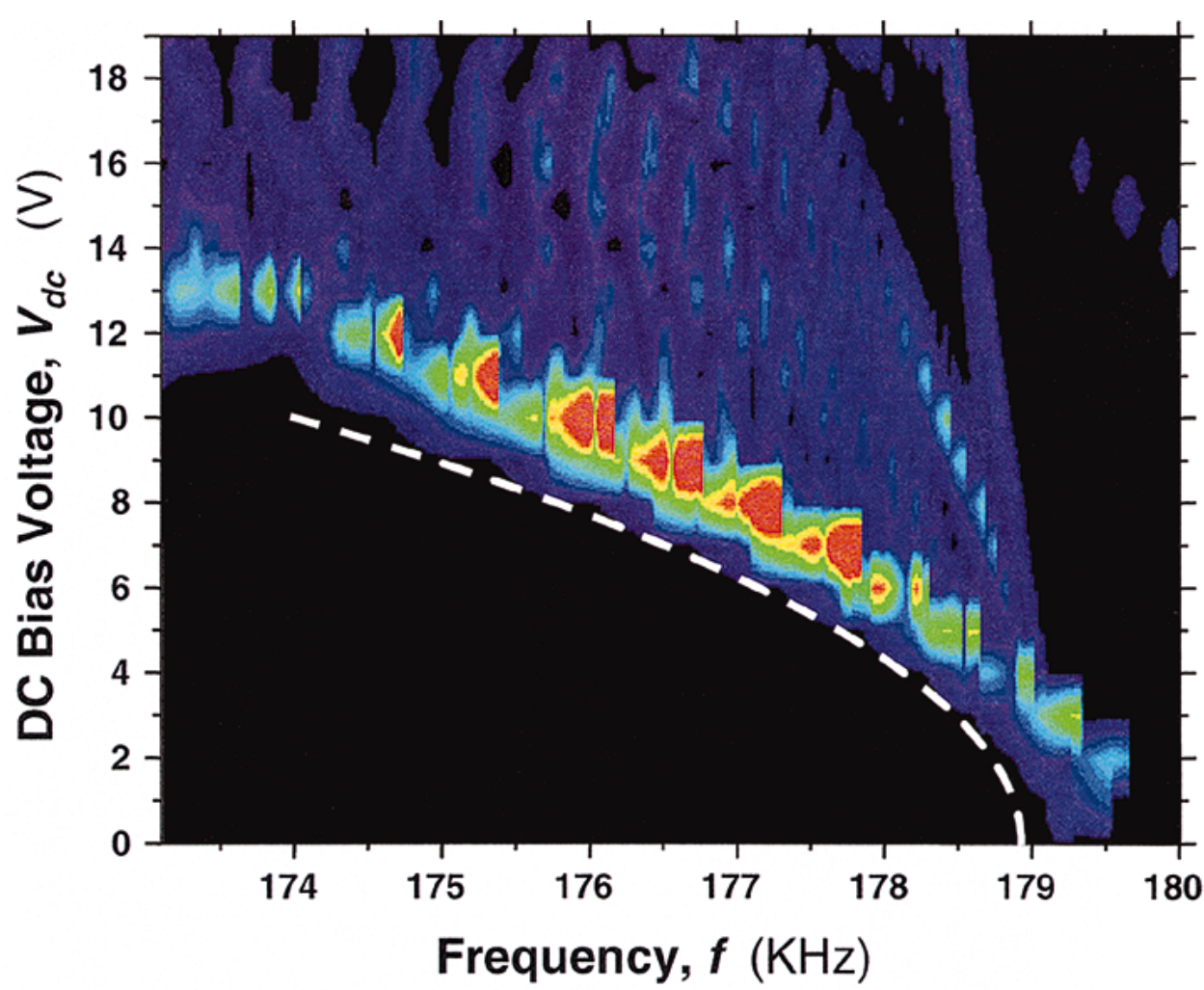

Fig. 5. A color map showing relative signal intensities measured at the lock-in amplifier operating in a $2 f$ mode. The dependence upon both the voltage $V_{\mathrm{dc}}$ and the frequency of the ac voltage $f$ are shown. The wavelength is tuned to the diffraction peak, namely $\lambda=1582 \mathrm{~nm}$. The dashed white line shows a fit to the measured lower bound frequency $f_{b}$ of the band.

fixed. We obtained similar behavior of this upper frequency with orthogonal polarization, and with another sample of similar design.

The rich and detailed structure of the frequency-dependent response $R$ observed in our experiments is not at present fully understood. Experimentally, Fig. 5 shows that $R$ is an oscillatory function of frequency, and has a relatively large magnitude close to the lower limit of the band. Theoretically the frequency dependence can be found from (6) and the density of states (which is determined by the dispersion relation). As discussed above, however, the simple linear theory is not adequate for predicting the steady-state response $A_{m}$. The experimentally observed oscillatory behavior indicates that nonlinear effects indeed dominate the dynamics of our system. To account for these observations, Lifshitz and Cross [15] have developed recently a theoretical model of the system presented in this work. Their analyses include the effects of nonlinear elasticity as well as nonlinear dissipation. By numerically integrating the nonlinear equations of motion, they obtain the response of the system to parametric drive. The calculated response successfully reproduces some of the features observed in the experiment, showing thus good qualitative agreement, however, further work is required to achieve also quantitative agreement.

\section{CONCLUSION}

Electrically tunable arrays offer unique prospects for optomechanical signal processing devices such as tunable filters and optical modulators. A particularly intriguing example is opto- mechanical spectral analysis of electrical waveforms. Consider an arbitrary electrical signal applied between the two comb electrodes of our device. Its Fourier components falling within the vibrational band (formed by the DC electrostatic coupling as described above) will parametrically drive the collective modes of the array. Each of these excited modes will result in a diffracted order with strength directly proportional to the respective Fourier component present within the applied waveform. Since each order is diffracted at a characteristic angle, continuous, real-time spectral analysis of the applied signal can be realized by using a photodetector array to collect the optical output at all angles. In principle, scaling the size of the resonant mechanical elements downward into the realm of NEMS (nano electromechanical systems) will permit extension of the operating frequency of such a analyzer to very high frequencies.

In conclusion, we have demonstrated the ability to induce and control collective modes of mechanical vibration within an array of micromechanical elements that, in effect, constitutes an artificial mesoscopic lattice. Further experimental and theoretical work will elucidate the rich dynamics of such systems, and will permit their optimization for novel micro opto-mechanical device applications.

\section{ACKNOWLEDGMENT}

The authors are grateful to K. Schwab for his assistance in sample fabrication and to R. Lifshitz for correcting an algebraic error in the manuscript. They also thank R. Lifshitz and M. Cross for helpful discussions. 


\section{REFERENCES}

[1] D. J. Bishop, "The rise of optical switching," Scientif. Amer., vol. 284, p. 88,2001

[2] S. G. Adams, F. M. Bersch, K. A. Shaw, and N. C. MacDonald, "Independent tuning of linear and nonlinear stiffness coefficients," J. Microelectromech. Syst., vol. 7, p. 172, 1998.

[3] C. T.-C. Nguyen, L. P. B. Katehi, and G. M. Rebeiz, "Micromachined devices for wireless communications," Proc. IEEE, vol. 86, p. 1756, 1998.

[4] O. Solgaard, F. S. A. Sandejas, and D. M. Bloom, "Deformable grating optical modulator," Opt. Lett., vol. 17, p. 688, 1992.

[5] D. E. Sene, V. M. Bright, J. H. Comtois, and J. W. Grantham, "Polysilicon micromechanical gratings for optical modulation," Sens. Actuators, Phys. A, vol. 57, p. 145, 1996.

[6] M. Ueda, T. Shiono, T. Ito, and K. Yokoyama, "High-efficiency diffractive micromachined chopper for infrared wavelength and its application to a pyroelectric infrared sensor," App. Opt., vol. 37, p. 1165, 1998.

[7] E. Buks and M. L. Roukes, "Stiction, adhesion energy, and the Casimir effect in micromechanical systems," Phys. Rev. B, vol. 63, p. 33402 , 2001.

[8] — " "Metastability and the Casimir effect in micromechanical systems," EuroPhys. Lett., vol. 54, p. 220, 2001.

[9] N. W. Ashcroft and N. D. Mermin, Solid State Physics: Saunders College, 1976.

[10] L. D. Landau and E. M. Lifshitz, Mechanics: Pergamon, 1976.

[11] A. H. Nayfeh and D. T. Mook, Nonlinear Oscillations. New York: Wiley, 1979

[12] N. W. McLachlan, Theory and Application of Mathieu Functions. New York: Dover, 1964

[13] J. W. Goodman, Introduction to Fourier Optics. New York: McGraw Hill, 1996.

[14] J. Callaway, Quantum Theory of the Solid State. New York: Academic, 1991.

[15] R. Lifshitz and M. C. Cross. Response of parametrically-driven nonlinear coupled oscillators with application to micro- and nanomechanical resonator arrays. [Online]. Available: http://xxx.lanl.gov/abs/cond$\mathrm{mat} / 0208394$

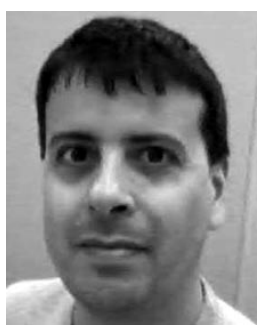

Eyal Buks received the B.Sc. degree in mathematics and physics from the Tel-Aviv University, Tel-Aviv, Israel, in 1991 and the M.Sc. and Ph.D. degrees in physics from the Weizmann Institute of Science, Israel, in 1994 and 1998, respectively. His graduate work concentrated on interference and dephasing in mesoscopic systems.

From 1998 to 2002, he worked at the California Institute of Technology (Caltech), Pasadena, as a Postdoctoral Scholar studying experimentally nanomachining devices. He is currently a Senior Lecturer at the Technion-Israel Institute of Technology, Haifa. His current research is focused on nanomachining and mesoscopic physics.

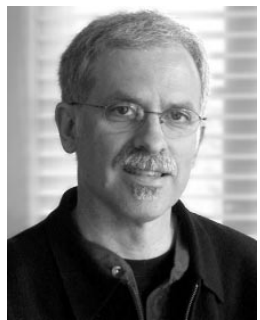

Michael L. Roukes received the B.A. degree with a double major of physics and chemistry from the University of California, Santa Cruz, in 1978 and the M.S. and Ph.D. degrees in physics from Cornell University, Ithaca, NY, in 1985.

He is currently a Professor of Physics, Applied Physics and Bioengineering at the California Institute of Technology (Caltech), Pasadena, where he has developed an extensive research program that utilizes nanomechanical and nanoelectronic devices as interfaces into the worlds of single spin dynamics and quantum information technologies, biomolecular detection and imaging, ultrasensitive mass spectrometry, and ultrasensitive calorimetry. $\mathrm{He}$ spent seven years as a Member of Technical Staff/Principal Investigator at Bell Communications Research before joining Caltech in 1992. In 2002, he was selected from the "Frontiers of Engineering" programs as a Lillian Gilbreth Lecturer by the National Academy of Engineering.

Dr. Roukes is a Fellow of the American Physical Society and a Co-Founder of Nanokinetics Corporation. 